\title{
Intraoperative radiation therapy in the treatment of early-stage breast cancer utilizing xoft axxent electronic brachytherapy Adam Dickler*1, Olga Ivanov ${ }^{2}$ and Darius Francescatti ${ }^{3}$
}

\author{
Address: ${ }^{1}$ Department of Radiation Oncology, Little Company of Mary Hospital, Evergreen Park, IL 60805, USA, ${ }^{2}$ Department of General Surgery, \\ Little Company of Mary Hospital, Evergreen Park, IL 60805, USA and ${ }^{3}$ Department of General Surgery, Rush University Medical Center, Chicago, \\ IL 60612, USA \\ Email: Adam Dickler* - atd22_99@yahoo.com; Olga Ivanov - olgaivanovmd@yahoo.com; Darius Francescatti - Dfrancescatti@gmail.com \\ * Corresponding author
}

Published: 2 March 2009

World Journal of Surgical Oncology 2009, 7:24 doi:10.1 186/1477-78/9-7-24

This article is available from: http://www.wjso.com/content/7///24

(c) 2009 Dickler et al; licensee BioMed Central Ltd.

This is an Open Access article distributed under the terms of the Creative Commons Attribution License (http://creativecommons.org/licenses/by/2.0), which permits unrestricted use, distribution, and reproduction in any medium, provided the original work is properly cited.
Received: 17 December 2008

Accepted: 2 March 2009

\begin{abstract}
Background: In an effort to overcome the barriers to $B C T$, alternative methods of delivering radiation therapy have been explored. APBI allows the radiation treatment to be accomplished in one week or less. XB is a form of balloon-based APBI that uses an electronic source generated by a mobile controller unit. Investigators have also explored IORT treatment that delivers a single fraction of radiation in the operating suite at the time of surgery.
\end{abstract}

Methods: We report on the first patient treated with $X B$ to deliver IORT.

Results: IORT treatment utilizing $X B$ is feasible and can be accomplished with a total procedure time of approximately 2 hours.

Conclusion: Further research on XB and other methods of IORT is needed to establish clinical efficacy and safety for patients with early-stage breast cancer.

\section{Background}

Several large randomized trials with extended follow-up have shown that breast conserving therapy (BCT) offers equivalent overall survival to modified radical mastectomy in patients with early stage breast cancer $[1,2]$. Studies have also shown that in certain parts of the United States, as few as $10 \%$ of eligible patients receive BCT $[3,4]$. Instead, some women are treated with mastectomy and others receive lumpectomy alone. This is especially common for women who live at an increased distance from radiation centers $[5,6]$. The logistical difficulties that accompany a 6-7 week course of whole breast external beam radiation (EBRT) are believed to be the main deterrent to patients receiving radiation therapy. Although radiation therapy is burdensome, it is an important component of BCT that cannot be safely omitted. EBRT has been shown to both decrease the risk of local recurrence and also to improve overall survival compared to surgery alone [7].

In an effort to overcome the barriers to $\mathrm{BCT}$, alternative methods of delivering radiation therapy have been explored. In contrast to standard EBRT, which treats the whole breast, accelerated partial breast irradiation (APBI) delivers radiation to the lumpectomy bed plus a $1-2 \mathrm{~cm}$ margin only. By decreasing the volume of treatment and increasing the daily fraction size of the radiation, treatment can be accomplished in one week rather than the standard 6-7 weeks.

The method of APBI with the longest published experience is multi-catheter interstitial brachytherapy. In this 
technique, several rows of catheter needles are placed around the lumpectomy bed and loaded with radiation sources. Multi-catheter interstitial brachytherapy has been associated with local recurrence rates of less than $5 \%$ at over 5-years follow-up and favorable cosmesis [8,9]. Unfortunately, this technique is difficult and requires significant expertise. As a result, multi-catheter interstitial breast brachytherapy is performed at relatively few centers in the U.S.

Balloon-based APBI methods were developed to simplify the brachytherapy procedure. The MammoSite ${ }^{\mathrm{TM}}$ (MS) brachytherapy applicator was the first balloon catheter to be developed for APBI. The catheter contains an inflation channel and a channel for the passage of an Iridium-192 (Ir-192) radiation source. It can be inserted through a single incision at the time of surgery or post-lumpectomy using ultrasound guidance. Initial results regarding cosmesis and local control using the MS catheter have been comparable to multi-catheter interstitial brachytherapy $[10,11]$.

Additional methods of balloon-based APBI are also being explored. A modified form of balloon-based brachytherapy called Xoft Axxent Electronic Brachytherapy ${ }^{\mathrm{TM}}$ (XB) received FDA clearance for the treatment of breast cancer in January, 2006. This device uses a mobile controller, which generates kilovoltage $(\mathrm{kV}) \mathrm{x}$-rays. This approach to APBI requires minimal shielding and thus has the potential to increase the number of settings in which radiation treatments can be offered. In addition, XB is not limited by rigorous radiation source regulations associated with other methods of APBI, which utilize radioisotope sources. The early results of a clinical trial to evaluate the performance and safety of XB in the outpatient treatment of early-stage breast cancer patients were recently presented at the American Society of Clinical Oncology (ASCO) Breast Cancer Symposium. Treatment with XB was found to be feasible and associated with minimal acute side effects [12].

Investigators have also explored delivering APBI in the operating room immediately after lumpectomy. Intraoperative radiation therapy (IORT) allows the patient to receive all her radiation in a single fraction before she awakens from surgery. Additional potential advantages include delivering the radiation before tumor cells have a chance to proliferate, performing the radiation under direct visualization at the time of surgery, and decreasing healthcare costs. Published results using IORT both as a tumor bed boost in conjunction with EBRT and as a primary treatment in APBI have shown favorable outcomes [13-15].
To date, XB has been utilized only in an outpatient setting to deliver APBI in 10 fractions over 5 days. We report on the first patient treated using XB to deliver IORT as part of an IRB approved single-institution trial.

\section{Patients and methods}

The patient is a 61-year-old woman who presented with an abnormal screening mammogram. It revealed an 8$\mathrm{mm}$ mass in the lower inner quadrant of the left breast. She had a core biopsy performed that showed a Grade 1, infiltrating ductal cancer that was estrogen and progesterone receptor positive.

The patient was seen in consultation by her surgeon and radiation oncologist. Radiation treatment options were explained to the patient, including the standard of care, 67 weeks of whole breast radiation therapy, and one week of outpatient APBI. An IRB approved protocol utilizing XB to deliver IORT was also discussed. The patient opted to enroll in the IORT protocol.

On September $3^{\text {rd }}$, 2008, the patient underwent a lumpectomy and sentinel lymph node biopsy. The tumor mass and margins of excision were sent for permanent section, and the identified sentinel lymph node was sent for frozen section. The pathology department reviewed the lymph node specimen at the time of surgery and informed the treating physicians that it was uninvolved by cancer.

At that point, additional breast tissue was removed posterior to the lumpectomy cavity down to the depth of the superficial pectoralis fascia to accommodate the chest wall shield. A pliable piece of lead was then temporarily placed over the chest wall to shield the ribs, lung, and heart, from scatter radiation. A cavity evaluation device (CED) was then inserted into the lumpectomy cavity through a small incision in the lateral breast and inflated with exactly 40 cc of saline (Figure 1). The conformity of the CED to the surrounding breast tissue was then evaluated under direct visualization. Conformance was considered to be acceptable if less than $10 \%$ of the tissue immediately surrounding the breast tissue was composed of fluid or air and the balloon was tightly opposed to the lumpectomy cavity. Conformance was found to be inadequate, so an additional $10 \mathrm{cc}$ of saline was inserted into the balloon. It was found that conformance was still less than ideal, so an additional $10 \mathrm{cc}$, or at total of $60 \mathrm{cc}$, was inserted into the balloon.

Since a CED inflated to $60 \mathrm{cc}$ was found to be the proper size, a $4 \times 5 \mathrm{~cm} \mathrm{XB}$ balloon catheter kit was then opened by the OR staff. The CED was then deflated and removed and a XB was then inserted in place of the CED. If a $40 \mathrm{cc}$ balloon were to be used, a $3 \times 4 \mathrm{~cm}$ catheter kit would have been opened. The physicist then began calibration of 


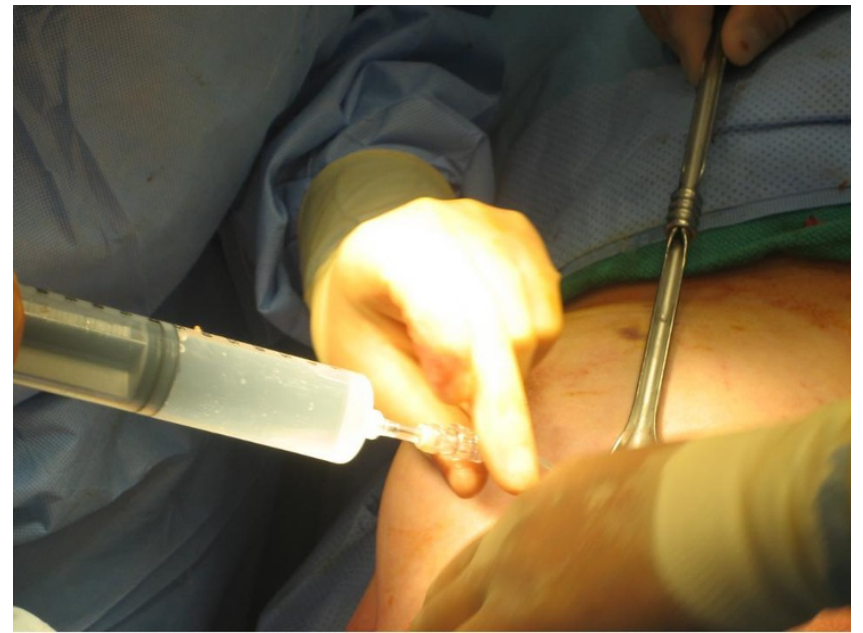

Figure I

A CED is inserted through a small incision in the lateral breast and inflated with exactly $40 \mathrm{cc}, 50 \mathrm{cc}, 60$ cc, or 70 cc.

the XB controller. Radiation plans for a $40 \mathrm{cc}, 50 \mathrm{cc}, 60 \mathrm{cc}$, and 70 cc balloon were previously developed by CT scanning the XB catheter inflated with saline to the 4 sizes in a water phantom. These plans were then loaded onto USB drives. At the completion of calibration, which typically takes approximately 15 minutes, the physicist then loaded the corresponding radiation plan onto the controller unit.

During machine calibration, retention sutures were placed in the breast tissue superficial to the inflated balloon (Figure 2). The retention sutures serve to build-up the subcutaneous tissue to potentially increase the balloon-to-skin distance and to more closely approximate the balloon to the surrounding breast tissue. An intraoperative ultrasound was then performed to evaluate the balloon-to-skin distance and the degree of air and/or fluid in the breast tissue surrounding the balloon. For the purposes of this protocol, a minimum of $1-\mathrm{cm}$ balloon to skin distance is required. If the patients are found to have $\geq 0.7-\mathrm{cm}$ and $<1.0-\mathrm{cm}$ of balloon-skin-distance they are offered outpatient balloon-based ABPI. If patients have a balloon-skin-distance of $<0.7-\mathrm{cm}$, balloon-based brachytherapy is aborted. If patients are found to have large air pockets surrounding the balloon on ultrasound, the balloon is inflated by $10 \mathrm{cc}$ to the next largest size and re-evaluated via ultrasound.

The patient was found to have $1.6-\mathrm{cm}$ balloon-to-skin distance and no air pockets surrounding the balloon, so the decision was made to proceed with IORT (Figure 3). A second sterile drape was then placed over the operative field, so that the radiation therapy could be delivered without

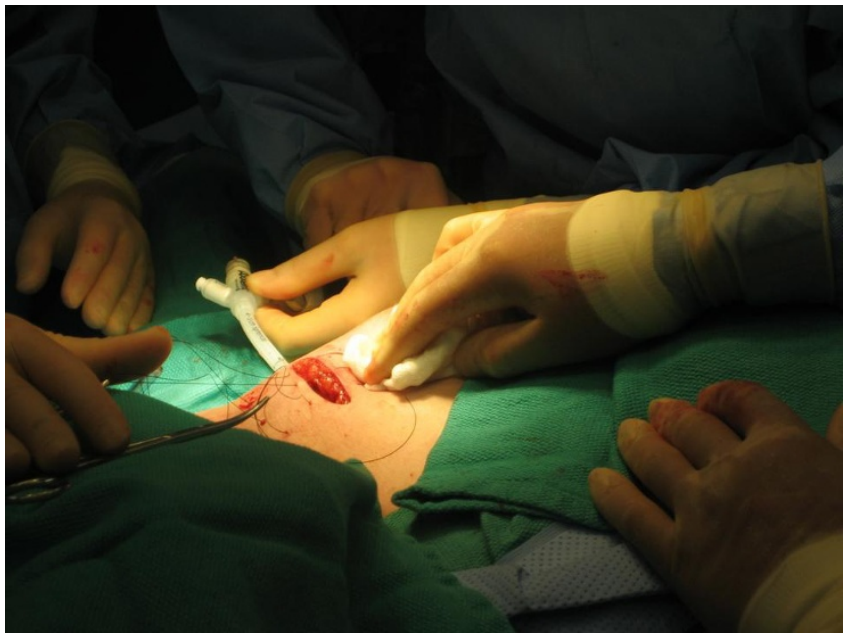

Figure 2

Retention sutures are placed to potentially increase the balloon-to-skin distance and to more closely approximate the balloon to the surrounding breast tissue.

contaminating the field. The catheter end was passed through a hole in the drape and a FlexiShield ${ }^{\mathrm{TM}}$ (FS) was placed on top of the drape (Figure 4). The FS is a leadequivalent material, which serves to decrease transmission to the patient and hospital staff.

\section{Results}

The radiation oncologist then broke scrub, attached the $\mathrm{XB}$ controller to the catheter, and inserted the radiation source into the balloon. The radiation therapy was then

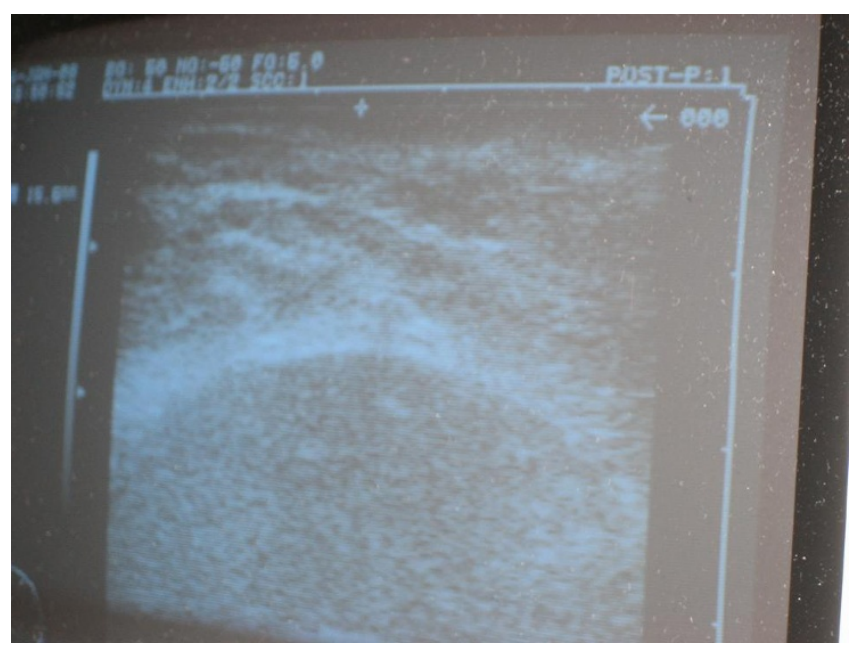

Figure 3

Ultrasound image showing a balloon-skin-distance of I.6-cm and no evidence of air pockets surrounding the balloon. 


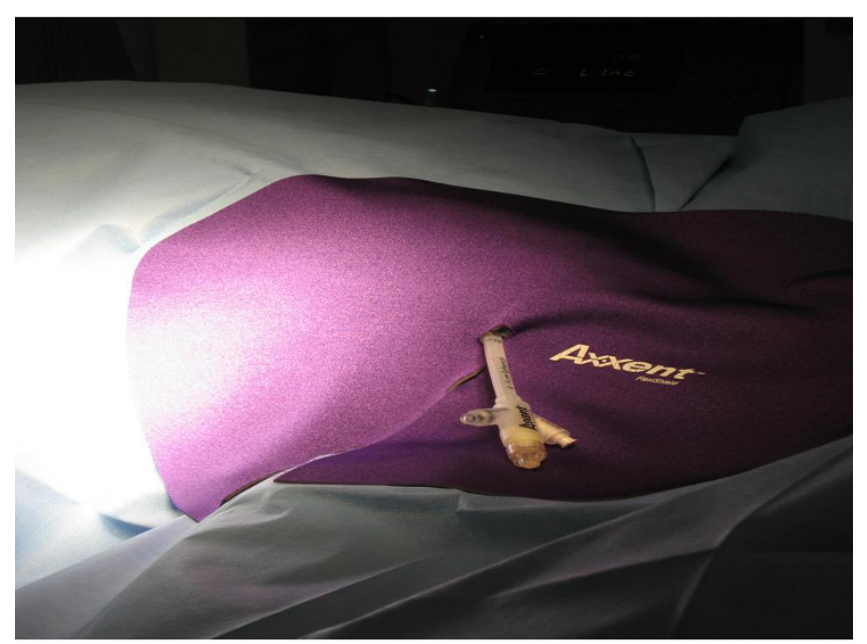

Figure 4

A sterile drape is placed over the operative field and a FlexiShield $^{\mathrm{TM}}$ is placed on top of the drape to minimize radiation transmission.

initiated. A total of $20 \mathrm{~Gy}$ to the balloon surface was delivered in approximately 20 minutes (Figure 5). The anesthesiologist, surgeon, and radiation oncologist remained in the room during the radiation delivery either wearing lead aprons or standing behind a mobile radiation shield. At the completion of the radiation, the balloon and temporary chest wall shield were removed and the incisions were closed. The duration of the entire procedure including lumpectomy, sentinel lymph node biopsy, balloon catheter placement, radiation therapy, and closing the incisions was approximately 2 hours.

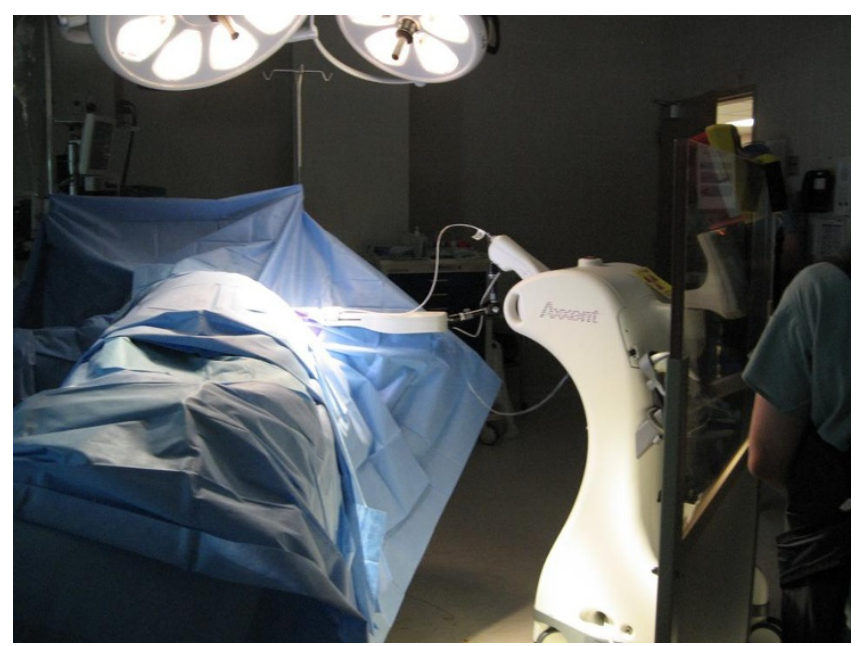

\section{Figure 5}

A radiation dose of 20 Gray to the balloon surface is delivered in approximately $\mathbf{2 0}$ minutes.
Margin status was assessed by permanent section after the completion of the surgery. Negative microscopic margins are required in the protocol for treatment with IORT alone. Patients who are found to have microscopically positive margins of excision are offered re-excision and whole breast EBRT. The patient was found to have margins of excision of over 2-mm

\section{Discussion}

$\mathrm{XB}$ is a novel form of balloon-based APBI, which uses an electronic source to generate $\mathrm{kV} x$-rays. This form of APBI eliminates the need for an HDR afterloader device, heavy shielding, and regulations associated with radiosotope handling and disposal. In addition, XB increases the number of settings in which radiation can be performed. Early clinical reports have shown this technique to be feasible and associated with minimal acute toxicity [12]. Also, XB has been compared to MS (a more established form of balloon-based APBI) and found to offer equivalent target volume coverage with increased normal tissue sparing [16]. To date and since FDA clearance, XB has only been used for outpatient treatment of breast and endometrial cancer. The mobile nature of the XB controller, as well as, the limited shielding requirements make XB a logical modality to be utilized for IORT in the treatment of early-stage breast cancer.

Delivering IORT treatment in a single session is not a new concept. Older IORT techniques involved cumbersome machinery or required custom-built heavily shielded operating suites. Other institutions have performed IORT by transferring patients from the operating room to the radiation oncology department during surgery.

Newer devices for the delivery of IORT utilize smaller, more mobile technology. Two modern mobile linear accelerators have been developed which use 4-6 MeV electrons to deliver a physical dose of approximately 21 Gy $[15,17]$. An additional device used to deliver IORT is the Intrabeam $^{\mathrm{TM}}$ (IB) device, which like XB, uses a $50 \mathrm{kV} \mathrm{x}-$ ray source. The IB device delivers $20 \mathrm{~Gy}$ at the surface of a solid spherical applicator and approximately $5 \mathrm{~Gy}$ at $1-\mathrm{cm}$ depth $[18,19]$. This is in contrast to XB IORT, which delivers $20 \mathrm{~Gy}$ at the surface of the balloon applicator and 9$10 \mathrm{~Gy}$ at $1-\mathrm{cm}$ depth. It is unknown whether the increased dose at 1-cm depth associated with XB will have a clinical benefit or possibly increase the risk of toxicity. XB and IB methods of IORT are compared in Table 1.

Long-term data regarding the safety and efficacy of IORT are not available. The TARGIT trial is a phase III prospective, randomized trial comparing single fraction IORT delivered with IB to conventional whole breast EBRT. Sixteen international institutions are enrolling patients in the trial. Eligible patients include patients $\geq 35$ years of age 
Table I: A comparison of IB and XB methods of IORT

\begin{tabular}{cccccc}
\hline & Source & Dose at Surface & Dose at I-cm Depth & Applicator Type & Treatment Time \\
\hline IB & $50 \mathrm{kV}$ x-rays & $20 \mathrm{~Gy}$ & $5 \mathrm{~Gy}$ & Solid Spherical & $20-45$ minutes* \\
\hline XB & $50 \mathrm{kV}$ x-rays & $20 \mathrm{~Gy}$ & $9-10 \mathrm{~Gy}$ & Balloon Catheter & $17-26$ minutes* \\
\hline
\end{tabular}

$\mathrm{IB}=$ Intrabeam $^{\mathrm{TM}}, \mathrm{XB}=\mathrm{Xoft}^{\mathrm{TM}}, \mathrm{kV}=$ kilovoltage, $\mathrm{Gy}=$ Gray.

* - Treatment time is dependant on applicator diameter used

with T1-T3, N0 tumors eligible for BCT. Patients with multi-focal or multi-centric lesions, clinically positive lymph nodes, extensive intraductal component, or invasive lobular cancers are not eligible for enrollment [19].

The results of the TARGIT trial will help determine whether IORT is an equivalent alternative to standard whole breast EBRT. If IORT methods, including $\mathrm{XB}$, are established as a standard treatment option, this may allow increased access to BCT, as well as, improved quality of life and decreased medical costs for patients with a diagnosis of early-stage breast cancer.

\section{Conclusion}

IORT utilizing XB is feasible and can be accomplished in a total procedure time of approximately 2 hours. To date, there are no long-term results utilizing this technique, and patients continue to be enrolled as part of an IRB approved single institution trial. Further research on XB and other methods of IORT is needed to establish clinical efficacy and safety for patients with early-stage breast cancer.

\section{Abbreviations}

BCT: Breast conserving therapy; EBRT: External beam radiation; APBI: Accelerated partial breast irradiation; MS: MammoSite; Ir-192: Iridium-192; XB: Xoft Axxent Electronic Brachytherapy; kV: kilovoltage; ASCO: American Society of Clinical Oncology; IORT: Intraoperative Radiation Therapy; CED: Cavity Evaluation Device; FS: FlexiShield $^{\text {TM }}$

\section{Consent}

Written consent for publication was obtained from the patient.

\section{Competing interests}

Dr. Dickler is on the Scientific Advisory Board for Xoft, Inc.

Dr. Francescatti is the Surgical Medical Director for Xoft, Inc.

\section{Authors' contributions}

$\mathrm{AD}$ was the treating radiation oncologist and developed the radiation portion of the technique. IO was the primary surgeon and helped to develop the surgical technique. DF was the assisting surgeon and helped to develop the surgical technique. All authors read and approved the final manuscript.

\section{References}

I. Veronesi U, Cascinelli N, Mariani L, Greco M, Saccozzi R, Luini A, Aguilar M, Marubini E: Twenty-year follow-up of a randomized study comparing breast-conserving surgery with radical mastectomy for early breast cancer. N Engl J Med 2002, 347:1227-I 232.

2. Fisher B, Anderson S, Bryant J, Margolese RG, Deutsch M, Fisher ER, Jeong $\mathrm{J}$, Wolmark $\mathrm{N}$ : Twenty-year follow-up of a randomized trial comparing total mastectomy, lumpectomy, and lumpectomy plus irradiation for the treatment of invasive breast cancer. N Engl J Med 2002, 34 I: I233-I24I.

3. Farrow DC, Hunt WC, Samet JM: Geographic variation the treatment of localized breast cancer. N Engl J Med 1992, 326: I097-II0I.

4. Dolan JT, Granchi TS, Miller CC 3rd, Brunicardi FC: Low use of breast conservation surgery medically indigent populations. Am J Surg 1999, I 78:470-474.

5. Athas WF, Adams-Cameron M, Hunt WC, Amir-Fazli A, Key CR: Travel distance to radiation therapy and receipt of radiotherapy following breast-conserving surgery. J Natl Cancer Inst 2000, 92:269-27I.

6. Hahn CA, Marks LB, Chen DY, Lind PA, Lind HM, Prosnitz LR: Breast conservation rates-barriers between tertiary care and community practice. Int J Radiat Oncol Biol Phys 2003, 55(5): I 196-I 199.

7. Clarke M, Collins R, Darby S, Davies C, Elphinstone P, Evans E, Godwin J, Gray R, Hicks C, James S, MacKinnon E, McGale P, McHugh T, et al: Effects of radiotherapy and differences in the extent of surgery for early breast cancer on local recurrence and I5year survival: an overview of the randomized trials. Lancet 2005, 366:287-2106.

8. Vicini F, Kestin L, Chen P, Benitez P, Goldstein NS, Martinez A: Limited-field radiation therapy in the management of early stage breast cancer. J Natl Cancer Inst 2003, 95:I205- I 210.

9. King TA, Bolton JS, Kuske RR, Fuhrman GM, Scroggins TG, Jiang XZ: Long-term results of wide-field brachytherapy as the sole method of radiation therapy after segmental mastectomy for T(is, I,2) breast cancer. Am J Surg 2000, I 80:299-304.

10. Vicini FA, Beitsch P, Quiet C, Keleher AJ, Garcia D, Snider HC Jr, Gittleman MA, Zannis VJ, Kuerer HM, Lyden M: Three-year analysis of treatment efficacy, cosmesis and toxicity by the American Society of Breast Surgeons (ASBS) MammoSite breast brachytherapy registry trial in patients treated with accelerated partial breast irradiation (APBI). IJROBP 2007, 69:S207.

II. Benitez PR, Keisch ME, Vicini F, Stolier A, Scroggins T, Walker A, White J, Hedberg $P$, Hebert M, Arthur D, et al.: Five-year results: the initial clinical trial of MammoSite balloon brachytherapy for partial breast irradiation in early-stage breast cancer. Am Surg 2007, 194:456-462.

12. Mehta VK, Dooley W, Griem KL, Dickler A, Haile K, Wazer DE, Kurtzman S, Chadha M, Steven RE, Modin SD, Elliott K: Early expe- 
rience with an electronic brachytherapy technique for intracavitary accelerated partial breast irradiation. Proceedings of the 2008 ASCO Breast Cancer Symposium 2008 [http:/l] www.asco.orASCO/

Abstracts $+\% 26+$ Virtual+MeetinAbstracts? \&vmview=abst detail vie $w \&$ conflD $=58 \&$ \&abstrac $\mathrm{tID}=404 \mid 4]$.

13. Vaidya JS, Walton L, Dewar J: Single dose targeted intraoperative radiotherapy (TARGIT) for breast cancer can be delivered as a second procedure under local anaesthetic. World J Surg Oncol 2006, 4:2.

14. Vaidya JS, Baum M, Tobias JS, Massarut S, Wenz F, Murphy O, Hilaris B, Houghton J, Saunders C, Corica T, et al:: Targeted intraoperative radiotherapy (TARGIT) yields very low recurrence rates when given as a boost. Int J Radiat Oncol Biol Phys 2006, 66: I335-8.

15. Intra M, Gatti G, Luini A, Galimberti V, Veronesi P, Zurrida S, Frasson A, Ciocca M, Orecchia R, Veronesi U: Surgical technique of intraoperative radiotherapy in conservative treatment of limitedstage breast cancer. Arch Surg 2002, I37:737-40.

16. Dickler A, Kirk MC, Seif N, Griem K, Dowlatshahi K, Francescatti D, Abrams RA: A dosimetric comparison of MammoSite highdose-rate brachytherapy and Xoft Axxent electronic brachytherapy. Brachytherapy 2007, 6:164-8.

17. Veronesi U, Orecchia R, Luini A, Gatti G, Intra M, Zurrida S, Ivaldi G, Tosi G, Ciocca M, Tosoni A, et al:: A preliminary report of intraoperative radiotherapy (IORT) in limited-stage breast cancers that are conservatively treated. Eur J Cancer 200I, 37(17):2178-2183.

18. Holmes DR, Baum M, Joseph D: The targit trial: targeted intraoperative radiation therapy versus conventional postoperative whole-breast radiotherapy after breast-conserving surgery for the management of early-stage invasive breast cancer (a trial update). Amer J Surg 2007, 194:507-5I0.

19. Vaidya JS, Tobias JS, Baum M, Wenz F, Kraus-Tiefenbacher U, D'souza D, Keshtgar M, Massarut S, Hilaris B, Saunders C, et al.: Targeted intraoperative radiotherapy: an innovative approach to partial breast irradiation. Sem Radiat Oncol 2005, I5:84-91.

Publish with Bio Med Central and every scientist can read your work free of charge

"BioMed Central will be the most significant development for disseminating the results of biomedical research in our lifetime. "

Sir Paul Nurse, Cancer Research UK

Your research papers will be:

- available free of charge to the entire biomedical community

- peer reviewed and published immediately upon acceptance

- cited in PubMed and archived on PubMed Central

- yours - you keep the copyright
BiolMedcentral 